\title{
Topography of Reward and Aversion Encoding in the Mesolimbic Dopaminergic System
}

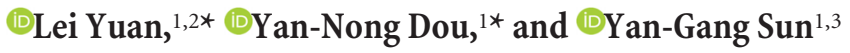 \\ ${ }^{1}$ Institute of Neuroscience, State Key Laboratory of Neuroscience, Center for Excellence in Brain Science \& Intelligence Technology, Chinese Academy of \\ Sciences, Shanghai 200031, China, ${ }^{2}$ University of Chinese Academy of Sciences, Beijing 100049, China, and ${ }^{3}$ Shanghai Center for Brain Science \\ and Brain-Inspired Intelligence Technology, Shanghai 201210, China
}

Dopamine (DA) neurons in the VTA play essential roles in adaptive motivated behavior, which requires rapid discrimination between positive and negative motivational signature. However, the precise functional DA circuitry processing reward and aversive information remain elusive. Here, we report that the encoding of reward and aversion by the DA system in the NAc is tightly associated with its anatomical location. By recording the dynamics of DA release with genetically encoded fluorescent DA sensor using in vivo fiber photometry in freely moving male mice, we found that the DA-sensor signal in the dorsomedial NAc shell and dorsolateral NAc shell were increased during rewarding events and decreased during aversive noxious events. In contrast, the release of DA in the ventromedial NAc shell was increased by both rewarding and aversive stimuli, whereas the DA-sensor signal in the central ventromedial NAc shell and ventrolateral NAc shell showed complex dynamics. Furthermore, the activity of DA fibers in different subregions of NAc measured with calcium sensor largely recapitulated the changes of DA-sensor signal in response to rewarding and aversive stimuli. In addition, correlation analysis showed that the response magnitude of DA-sensor or fibers significantly changed along the DV axis of the NAc. These results revealed the distinct role of the mesolimbic DA system in different subregions of NAc in encoding value and salience.

Key words: dopamine; NAc; salience; value

\section{Significance Statement}

Adaptive motivated behavior requires rapid discrimination between favorable and harmful events and is dynamically modulated by dopamine (DA) neurons in the VTA. However, the precise relationship between distinct DA circuitry and reward/aversion signal encoding is not well understood. Here, by recording the dynamics of DA release and the activity of DA fibers in each subregion of the NAc using in vivo fiber photometry in freely moving animals, we found that the DA system in the dorsomedial/ dorsolateral, ventromedial, and ventrolateral NAc shell plays different roles in encoding value and salience. These results extend our knowledge about how the mesolimbic DA system process motivational information at the circuitry level.

\section{Introduction}

Dopamine (DA) neurons in the VTA play an important role in reward processing and motivated behavior (Schultz et al., 1997;

Received Feb. 1, 2019; revised May 29, 2019; accepted June 5, 2019.

Author contributions: L.Y. and Y.-G.S. designed research; L.Y. and Y.-N.D. performed research; L.Y. and Y.-G.S. analyzed data; L.Y. and Y.-G.S. wrote the first draft of the paper; L.Y. and Y.-G.S. edited the paper; L.Y. and Y.-G.S. wrote the paper.

This work was supported by National Natural Science Foundation of China 61890952 and 31825013, Shanghai Municipal Science and Technology Major Project Grant 2018SHZDZX05, and Program of Shanghai Academic/Technology Research Leader 19XD1424200. Y.-N.D. was supported by China Postdoctoral Science Foundation Grant 2018M640426 and the Shanghai Super Postdoctoral Incentive Program. We thank Yan-Jing Zhu for technical support; Yu-Long Li (Peking University) for the gift AAV-expressing florescent dopamine sensor; and all laboratory members of Y.-G.S. for helpful discussion.

The authors declare no competing financial interests.

*L.Y. and Y.-N.D. contributed equally to this work.

Correspondence should be addressed to Yan-Gang Sun at yangang.sun@ion.ac.cn.

https://doi.org/10.1523/JNEUROSCI.0271-19.2019

Copyright $@ 2019$ the authors
Wise, 2004; Schultz, 2006; Cohen et al., 2012). DA neurons are thought to mainly encode value signal as they are excited by reward or its associated cues and inhibited by aversive events. Moreover, the firing rates of DA neurons are modulated by the error between the actual outcome and the expected outcome (Schultz et al., 1997). Therefore, DA neurons signal the prediction errors and play critical roles in the learning of approaching favorable stimuli or escaping from unfavorable events to enable subjects to maximize reward and minimize punishment. Although DA neurons were thought to be homogeneous in their coding property (Ungless et al., 2004; Fiorillo, 2013), a large body of literature indicates that the DA neurons are heterogeneous in both molecular identity and function (Matsumoto and Hikosaka, 2009; Bromberg-Martin et al., 2010; Lammel et al., 2011; Matsumoto et al., 2016; Morales and Margolis, 2017; Gantz et al., 2018). For example, some DA neurons were excited, rather than inhibited, by aversive stimuli (Bromberg-Martin et al., 2010), leading 
to the idea that at least some DA neurons encode salience rather than value signal. The diverse functions of subpopulations of DA neurons are related to their anatomical position and connections (Brischoux et al., 2009; Lammel et al., 2012). However, the precise DA circuitry that signals value and salience information remains largely elusive.

The mesolimbic DA system, defined as the DA pathway from the VTA DA neurons to the NAc, plays important roles in processing reward, motivating approaching behaviors, and positive reinforcement (Björklund and Dunnett, 2007; Salamone and Correa, 2012; Berridge and Kringelbach, 2015; Hamid et al., 2016; Howe and Dombeck, 2016; Parker et al., 2016; Schultz, 2016; Saunders et al., 2018). Additionally, the mesolimbic DA system also processes aversive information as DA release was evoked by some aversive stimuli (e.g., bitter taste, footshock, and tail pinch) as measured by microdialysis or fast-scan cyclic voltammetry techniques (Bassareo et al., 2002; Martinez et al., 2008; Anstrom et al., 2009; Badrinarayan et al., 2012; Budygin et al., 2012; McCutcheon et al., 2012). It is possible that the function of the mesolimbic DA system differs in different subregions of NAc. For example, aversive taste stimuli (quinine and saturated $\mathrm{NaCl}$ solutions) rapidly evoked DA release in the NAc core but, instead, had no effect in the NAc shell (Bassareo et al., 2002). However, different stimuli types have sometimes led to inconsistent responses. For example, aversive fear cue decreased DA transmission within the NAc core and increased DA level within the NAc shell (Badrinarayan et al., 2012), whereas noxious tail pinch increased DA level in the NAc core (Budygin et al., 2012). Therefore, it is unclear how different reward and aversive information is processed by different subcircuits of the mesolimbic DA system.

In this study, by taking advantage of the new genetically encoded fluorescent GPCR activation-based DA $\left(\mathrm{GRAB}_{\mathrm{DA}}\right)$ sensors (Sun et al., 2018), we measured extracellular DA dynamics in distinct NAc subregions by in vivo fiber photometry during series of rewarding and aversive events in freely moving animals. We further confirmed the diverse representation of reward and aversion by the DA system in different NAc subregions, by examining the calcium activity of VTA DA projections in each region. Finally, by correlation analysis between the change of DA sensor/ fibers signal and corresponding sampling location, we explored the functional topography of value and salience encoding by the mesolimbic DA system.

\section{Materials and Methods}

\section{Animals}

WT (C57BL/6) male mice ordered from the Shanghai laboratory animal center were used for recording the dynamics of DA release. Dopamine transporter (DAT)::IRES-Cre knockin mice (referred to as DAT-Cre mice hereafter; JAX006660, RRID:IMSR_JAX:006660) were obtained from The Jackson Laboratory and crossed with WT C57BL/6 mice. Only male heterozygous DAT-Cre mice were used for recording the activities of DA fibers. Mice 8-12 weeks of age received virus injections for the fiber photometry recording. All the mice were housed in stable conditions with ad libitum access to food and water under a $12 \mathrm{~h}$ lightdark cycle (light off from 7:00 P.M. to 7:00 A.M.). All animal studies and experimental procedures were approved by the Animal Care and Use Committee of the Institute of Neuroscience, Chinese Academy of Sciences.

\section{Surgeries for viral delivery and optic fiber implantation}

Surgery was performed as previously described (Yuan et al., 2018). Before the surgical procedures, animals were anesthetized by an intraperitoneal injection of $1 \%$ pentobarbital sodium (dissolved in saline, 100 $\mathrm{mg} / \mathrm{kg}$ ), and then placed on the stereotaxic apparatus (Stoelting). To express fluorescent DA-sensor in each NAc subregion, AAV9-hSyn-DA- sensor $4.4\left(6 \times 10^{12}\right.$ genome copies $\left.(\mathrm{gc}) / \mathrm{ml}\right)$ was injected into the dorsomedial NAc shell (dmNAc) (AP $1.34 \mathrm{~mm}$, ML $0.65 \mathrm{~mm}$, and DV -4.1 $\mathrm{mm}$ relative to bregma), central ventromedial NAc shell (ceNAc) (AP $0.98 \mathrm{~mm}, \mathrm{ML} 1.3 \mathrm{~mm}, \mathrm{DV},-4.6 \mathrm{~mm}$ ), ventromedial NAc shell (vmNAc) (AP $1.54 \mathrm{~mm}$, ML $0.5 \mathrm{~mm}, \mathrm{DV},-4.5 \mathrm{~mm}$ ), dorsolateral NAc shell (dlatNAc) (AP $1.1 \mathrm{~mm}$, ML $1.7 \mathrm{~mm}, \mathrm{DV},-4.3 \mathrm{~mm}$ ), and ventrolateral NAc shell (vlatNAc) (AP $1.1 \mathrm{~mm}, \mathrm{ML} 1.7 \mathrm{~mm}, \mathrm{DV},-4.6 \mathrm{~mm}$ ), respectively. To express DA-insensitive control sensors in the NAc lateral shell, AAV9hSyn-DA-sensor-mut $\left(6.5 \times 10^{12} \mathrm{gc} / \mathrm{ml}\right)$ was injected into the dlatNAc and vlatNAc. To specifically express the fluorescent calcium indicatorGCaMP6s in VTA DA neurons, AAV5-hSyn-DIO-GCaMP6s $\left(4.5 \times 10^{12}\right.$ $\mathrm{gc} / \mathrm{ml}$, Shanghai Taitool Bioscience) was injected into the VTA (AP $-3.35 \mathrm{~mm}$, ML $0.45 \mathrm{~mm}$, DV $-4.25 \mathrm{~mm}$ ) of DAT-Cre mice. The virus was injected with a glass pipette (tip diameter $20-30 \mu \mathrm{m}$ ) at a rate of $0.05-0.1 \mu \mathrm{l}$ per minute $(0.3-0.4 \mu \mathrm{l}$ per site) unilaterally into left NAc subregions or VTA.

Following the adeno-associated virus (AAV) infusion, an optic fiber (diameter, $200 \mu \mathrm{m}$; NA, 0.37) was implanted 0.05-0.15 $\mathrm{mm}$ above the coordinates in each NAc subregion mentioned above for subsequent fiber photometry recording. Tissue gel (Kwik-Cast Sealant) first was added to the surgical hole to prevent contact between the dental cement and brain tissue. Then, gel (3M Vetbond Adhesive) was applied to the dry skull surface, and dental cement was applied to cover the skull surface.

\section{Fiber photometry recording}

Response to rewarding and aversive taste stimuli. Following viral injection, mice were allowed to recover 3-4 weeks. Then, mice were daily handled for $5 \mathrm{~min}$ and water-restricted for $3 \mathrm{~d}$. Water ( $1.5 \mathrm{ml}$ for each mouse) was daily applied with a dish (diameter, $5.8 \mathrm{~cm}$ ), which was put into the home cage 1 week beforehand, to maintain mice at $85 \%-90 \%$ of ad libitum weight (Patriarchi et al., 2018). During the photometry recording experiment, cagemates were moved to a holding cage, and the mouse for the experiment was put back to its home cage to freely explore for $3 \mathrm{~min}$ while the implanted optical fiber was connected to an external patch cord. Each taste stimuli (water, $1 \mathrm{~mm}$ quinine in water, or $5 \%$ sucrose water) was applied with the dish at $50 \mu \mathrm{l}$ per trial for 3 times in random order. Care was taken to prevent mice from visualizing the stimuli application to avoid motivational cue-induced predicted DA release. Each taste stimulus was applied at an interval of $\sim 60 \mathrm{~s}$. By carefully monitoring mice's behavior, the onset of each liquid consumption was tagged by triggering TTL signal (Isolated Pulse Stimulator Model 2100, A-M Systems), which was synchronously output to the fiber photometry system. The TTL signal and fluorescence signal were recorded simultaneously at $1000 \mathrm{~Hz}$ with F-scope-G-2 (Biolink Optics). To examine the DA-sensor signal within liquid consumption, animal behavior was videotaped for some mice expressing DA-sensor in the dlatNAc and vlatNAc. The laser power was $\sim 15 \mu \mathrm{W}$ at the tip of implanted optic fiber. The system noise was recorded by disconnecting the tip of photometry fiber from implanted fiber and blocking any optic input. Data were analyzed using a custom-written MATLAB code.

Response to noxious footshock and tail pinch. Mice were placed on a footshock device (AniLab) or an open field and habituated for 3 min while the implanted optic fiber was connected to an external patch cord. Unexpected footshocks $(0.6 \mathrm{~mA}, 1 \mathrm{~s})$ or tail pinch using a clip (5-10 s) was delivered for $4-8$ times with an interval of $30-60 \mathrm{~s}$. The start or stop time of each footshock and tail pinch stimuli was tagged by TTL signal, as mentioned above. All the mice in the dmNAc, ceNAc, and vmNAc groups first underwent the liquid consumption trials, then the tail pinch, and finally the footshock, in different days to minimalize the possible effect of the previous stimulus on subsequent experiments. The mice expressing DA-sensor in the dlatNAc and vlatNAc first underwent the liquid consumption trials (12 of 20 mice) and then the footshock (all the 20 mice), whereas the mice expressing DA-sensor-mut in the dlatNAc and vlatNAc only underwent the footshock challenge to rule out the movement artifacts possibility.

\section{Fiber photometry data analysis}

The onset or offset of event stimuli was identified by a custom-written code. Fluorescence values were low-pass filtered at $2 \mathrm{~Hz}$ using a fourth- 
order Butterworth filter with zero-phase distortion and subtracted the noise signal of the recording system. The resulting values were aligned to event onset or offset, and corresponding fluorescence values in each stimulus were derived. The dynamics of fluorescence in each stimulus were calculated by the following:

$$
\Delta F / F=\left(F-F_{0}\right) / F_{0}
$$

$F$ indicates fluorescence values in each time point, and $F_{0}$ indicates the median of fluorescence values during baseline window $(-5$ to $-3 \mathrm{~s}$ for taste stimuli, -2.5 to $0 \mathrm{~s}$ for footshock stimulus, and -5 to $0 \mathrm{~s}$ for tail pinch stimulus). To quantify the change of fluorescence values across stimulation period, the event windows were defined (2-7 s for liquid consumption, $0-2.5 \mathrm{~s}$ after the onset of footshock, $1-5 \mathrm{~s}$ after the onset or offset of a tail pinch). The averaged fluorescence changes in each baseline and event window were calculated and compared. To directly visualize the change of DA sensor-signal in different stimuli or its association with sampling sites for individual animals, the joint response was represented by a scatter diagram.

\section{Histology}

Mice were anesthetized with overdosed $1 \%$ pentobarbital sodium and perfused with saline followed by $4 \%$ PFA (Sigma) in 0.01 м PBS. The brain was carefully removed and postfixed in 4\% PFA for an extra $4 \mathrm{~h}$, after which it was kept in $30 \%$ sucrose solution in $0.01 \mathrm{M} \mathrm{PBS} 4^{\circ} \mathrm{C}$ for $2 \mathrm{~d}$. Free-floating coronal sections $(40 \mu \mathrm{m})$ prepared with a cryostat (Leica Microsystems, CM 1950) were used for immunofluorescent staining. For identifying the expression of DA-sensor, DA-sensor-mut, and GCaMP6s, sections were blocked in 5\% normal donkey serum in PBST $(0.3 \%$ Triton $\mathrm{X}-100$ in $0.01 \mathrm{~m} \mathrm{PBS})$ for $30 \mathrm{~min}$ at room temperature, followed by incubation with primary antibody (rabbit anti-GFP, 1:500, Thermo Fisher Scientific, catalog \#A-11122; RRID:AB_221569) in PBST (with $1 \%$ normal donkey serum) at $4^{\circ} \mathrm{C}$ overnight and secondary antibody (AlexaFluor-488-donkey anti-rabbit, 1:400, Jackson ImmunoResearch Laboratories, catalog \#711-545-152; RRID:AB_2313584) in PBST (with $0.5 \mu \mathrm{g} / \mathrm{ml}$ DAPI) at room temperature for $2 \mathrm{~h}$. Photomicrographs were taken with the Olympus VS120 microscope and processed using ImageJ software (National Institutes of Health, RRID:SCR_003070).

\section{Experimental design and statistical analysis}

We did not predetermine sample sizes by statistical tests, but our sample sizes were similar to previously published studies. The sample size of each experiment is provided in Results. The data are expressed as mean \pm SEM. The statistical analyses were performed using Prism 6, and the confidence level was set to 0.05 . $p$ values less than 0.001 are reported as $p<0.001$, and all statistical parameters are reported in Results. Two-way repeated-measures ANOVA was used to analyze the significance of the interaction between region versus taste stimuli or period factors for fluorescence changes. Bonferroni-corrected post hoc comparisons were performed to compare fluorescence values between different periods or taste stimuli in individual regions. Pearson's correlation test was used to test the significance of the correlation between fluorescence changes and sampling location.

\section{Results}

\section{Subregion-specific changes of DA release during rewarding and aversive taste stimuli}

To examine the dynamics of DA-sensor signal in each NAc subregion, we injected AAV-expressing fluorescent DA-sensor (AAV-hSyn-DA-sensor 4.4) and implanted optic fiber into the dmNAc, ceNAc, or vmNAc in WT mice (Fig. $1 A, B$ ), respectively. To test the effect of rewarding and aversive stimuli on DA-sensor signal, water, bitter water (1 mM quinine), or sweet water $(5 \%$ sucrose) was randomly delivered to the water-restricted mice (Fig. 1C). The fluorescence of DA-sensor was collected by in vivo fiber photometry recording. Robust changes of fluorescent signal of DA-sensor were evoked when the mice were approaching the liquid (Fig. 1C). We first analyzed the dynamics of DA-sensor signal across water drinking for individual animals (Fig. 1D) and found that the DA-sensor signal in all subregions was increased significantly during water consumption (Fig. $1 E$ ). This result is consistent with the important roles of the limbic DA system in processing rewarding signal.

To test whether the DA-sensor signal would also be uniformly increased or decreased by aversive taste across all regions, we averaged the dynamics of DA-sensor signal during water drinking and bitter water drinking for different animals. Strikingly, we found that the elevated signal quickly decreased to the basal level after bitter water consumption in the dmNAc and ceNAc but not vmNAc (Fig. 1F). The quantification analysis showed that the response of DA-sensor signal to aversive bitter water was significantly smaller than that to water in the dmNAc and ceNAc (Fig. $1 G$ ). To rule out the possibility that the differential response was caused by a big decrease only in a subgroup of animals, we compared the response with two stimuli for individual mice and found that water evoked a bigger increase of DA-sensor signal in nearly all the mice for the dmNAc and ceNAc but not the vmNAc group (Fig. $1 H$ ). These results suggest that DA system in the dmNAc and ceNAc might encode value signal. We next asked whether the DA system in these areas could discriminate value magnitude by comparing the DA-sensor signal change caused by water/bitter water and sweet water (Fig. $1 F$ ). We found no significant difference in DA-sensor signal change between water and sweet water stimuli for all groups (Fig. $1 G$ ), and that the distribution of joint response to two stimuli was symmetrical for all groups (Fig. $1 H$ ), whereas sweet water elicited larger response than bitter water in the dmNAc and ceNAc (Fig. 1G). Collectively, these results suggest that the DA system encoded rewarding and aversive taste stimuli in a subregion-dependent manner.

\section{Topographic representation of nociceptive information in the NAc measured with DA-sensor}

Given the differential DA-sensor signal during aversive ingestion in distinct NAc subregions, we hypothesized that other aversion stimuli would also decrease the release of DA in the dmNAc and ceNAc, and increase the release in the vmNAc. To directly test this idea, we applied unexpected noxious footshock to the animals and recorded the dynamics of DA-sensor signal using the fluorescent DA-sensor simultaneously in each subregion of NAc (Fig. 2A). As expected, we observed that the noxious footshock decreased the fluorescence of DA-sensor in the dmNAc and increased the fluorescence signal of DA-sensor in the vmNAc (Fig. $2 B$ ). The response was robust in individual animals (Fig. 2C). In contrast, we found that the DA-sensor signal in the ceNAc was increased during footshock (Fig. $2 B, C$ ). The averaged dynamics of DA-sensor signal and quantitative analysis showed that the aversive footshock significantly decreased the DA-sensor signal in the dmNAc but increased the signal in the ceNAc and vmNAc (Fig. 2D,E).

To further elucidate the encoding of aversion by the DA system in each subregion of NAc, we tested the change of DA-sensor signal in response to another unexpected noxious stimulus, tail pinch (Fig. 2A). Consistently, the DA-sensor signal decreased in the dmNAc and increased in the vmNAc after tail pinch (Fig. $2 F$ ). Averaged dynamics and quantitative changes of the fluorescence signal of DA-sensor also revealed the opposite DA-sensor signal (Fig. 2G), although the change was not significant in the vmNAc. Meanwhile, unlike the increased change in response to footshock, the DA-sensor signal was conversely decreased in the ceNAc (Fig. $2 F, G)$. The inconsistent responses to two aversive noxious events in the same group of animals suggest that the DA system in the ceNAc encodes the mixture of value and salience. As the termi- 
A
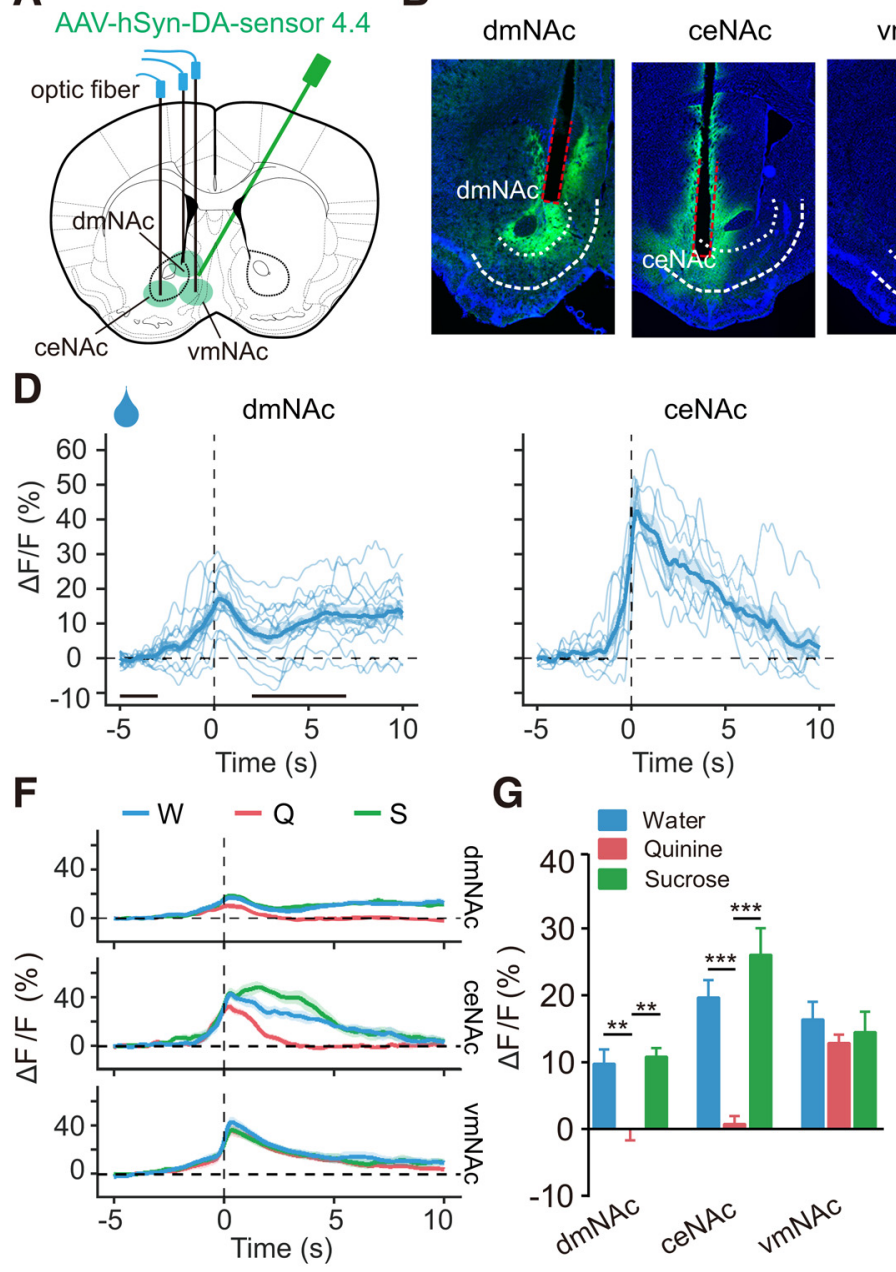

dmNAc

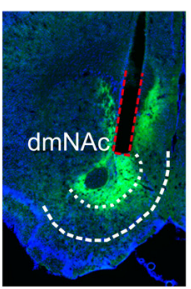

ceNAc

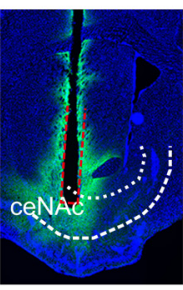

ceNAc

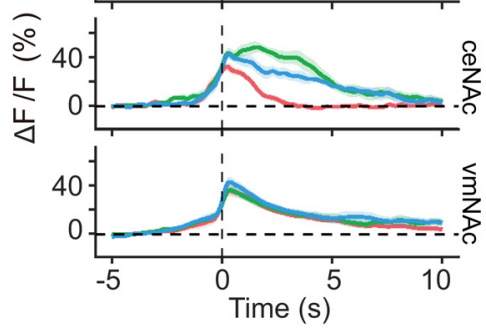

G

H
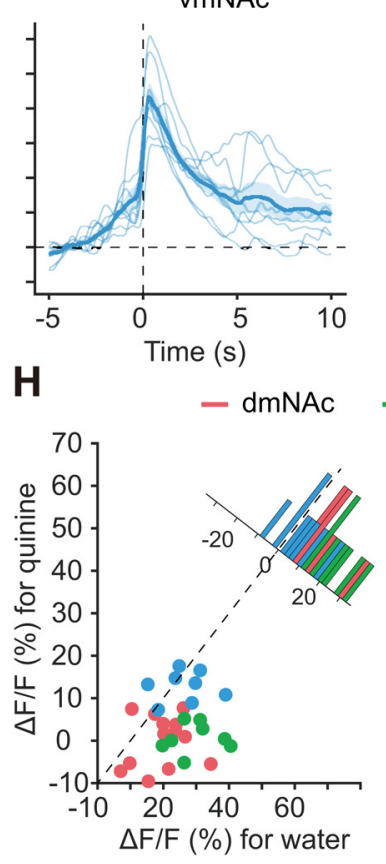
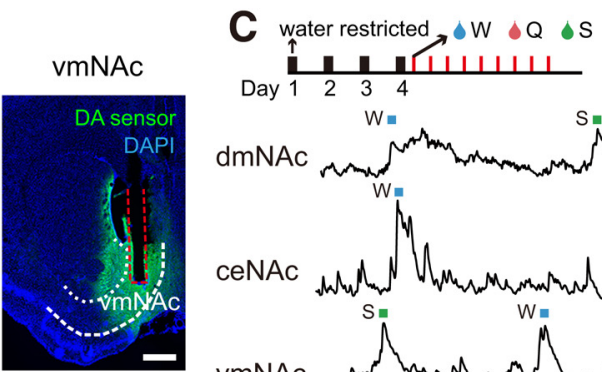

dmNAc

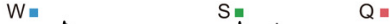

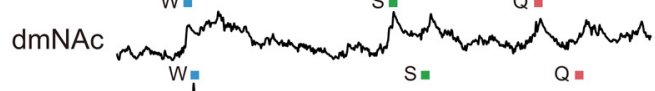

ceNAc

vmNAc

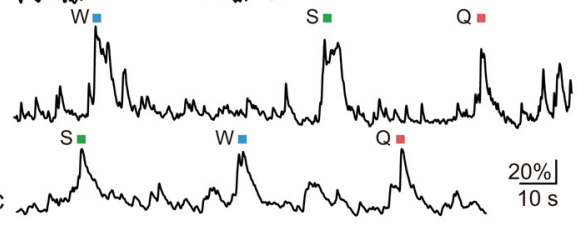

vmNAc

E
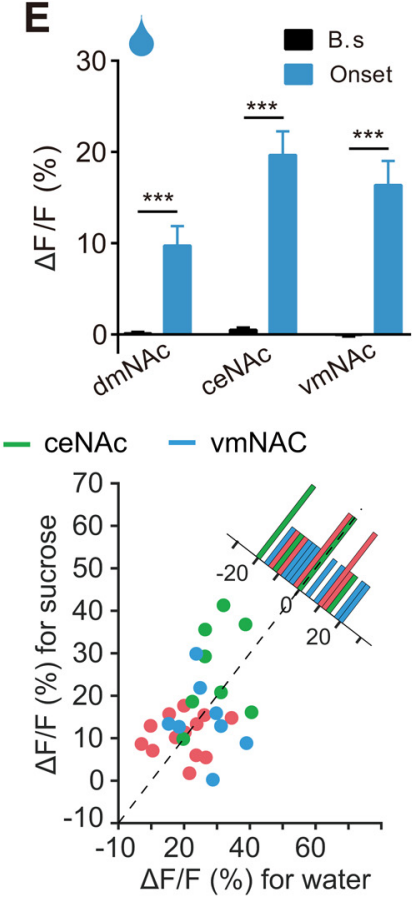

Figure 1. Different dynamics of DA-sensor signal during consumption of appetitive and aversive liquid in distinct NAc subregions. A, Schematic of virus injection and optic fiber implantation in WT animals. B, Example recording sites in each subarea. Dashed red line outlines the track of fiber optic. Scale bar, $500 \mu \mathrm{m}$. C, Schematic of liquid delivery and representative photometry traces simultaneously recorded in each subregion. W, Water; $Q$, water with quinine; $S$, water with sucrose. Colored bars above the example trace represent the onset of liquid consumption. $\boldsymbol{D}$, Fluorescence change of DA-sensor across water consumption in dmNAc group (left, $n=13$ mice), ceNAc group (middle, $n=8$ mice), and vmNAc group (right, $n=8$ mice). Thin lines indicate the response in individual animals. Black bars represent the baseline period $(-5$ to $-3 s, B . s)$ and onset period $(2-7 \mathrm{~s})$ used for quantification analysis. $\boldsymbol{E}$, Comparison of the averaged values of DA-sensor fluorescence during B.s and onset period for each group (two-way ANOVA revealed region $\times$ period interaction, $F_{(2,52)}=4.417, p=0.0169$; Bonferroni post hoc analysis, ${ }^{* * *} p<0.001$ between B.s and onset period for all groups). $\boldsymbol{F}$, Comparison of averaged fluorescence change in response to $W, S$, and $Q$ consumption in each subarea. $G$, Quantitative comparison of the response to different liquid consumption for dmNAc group (two-way ANOVA revealed region $\times$ taste interaction, $F_{(4,78)}=6.063, p=0.0003$; Bonferroni post hoc analysis, ${ }^{* *} p=0.0057$ between water and quinine and ${ }^{* *} p=0.0016$ between sucrose and quinine for dmNAcregion, ${ }^{* * *} p<0.001$ between water and quinine or between sucrose and quinine for ceNAc region, and $p>0.64$ for other comparisons). $\boldsymbol{H}$, Comparison of the DA-sensor signal change during the W and Q (left) or $S$ (right) consumption for individual animals. Corner histogram represents the distribution of differential response obtained by subtracting quinine/sucrose response from water response. Error bar and shadows represent SEM.

nation of aversive stimuli was found to be rewarding and DA was required in this process (Gerber et al., 2014), we also examined the change of DA-sensor signal after the offset of tail pinch. We found that the fluorescence signal of DA-sensor in the dmNAc and vmNAc elevated significantly during the relief of noxious stimuli (Fig. $2 \mathrm{H}$ ), further suggesting the coding possibility of value and salience by the DA system in the respective subregion. Finally, to rule out the possibility that the differential change of DA-sensor signal in different stimuli was mainly contributed by a subgroup of animals, we compared the response in two aversive events for individual animals and found dominant decrease in fluorescence signal of DA-sensor in both events for the dmNAc group, main increase in fluorescence signal of DA-sensor for vmNAc group, and opposite change for the ceNAc group (Fig. 2I). These results revealed that noxious footshock and tail pinch stimuli uniformly inhibited the DA release in the dmNAc and en- hanced the DA release in the vmNAc but evoked the opposite response in the ceNAc.

\section{The release of DA was differentially regulated by aversive} stimulus in the dlatNAc and vlatNAc shell

To get a full picture of the representation of reward and aversion by NAc DA system, we next performed recordings in the lateral shell of NAc (latNAc). As our results indicated that the DA release was differentially affected by aversive stimuli in the dmNAc and vmNAc, we targeted the dlatNAc and vlatNAc in separate cohorts of mice (Fig. 3A). First, we tested the dynamics of DA-sensor signal during the delivery of liquid taste stimuli to waterrestricted mice and found that liquid consumption evoked increase of DA-sensor signal (Fig. 3B). To test whether aversive taste stimuli affected the DA-sensor signal, we statistically compared the change of DA-sensor signal during bitter water, water, 
A
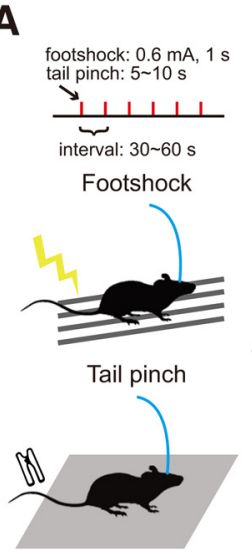

F

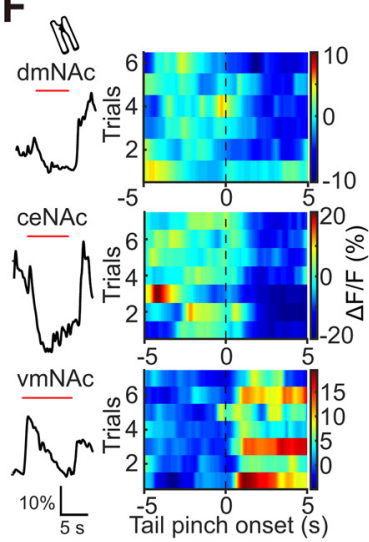

B
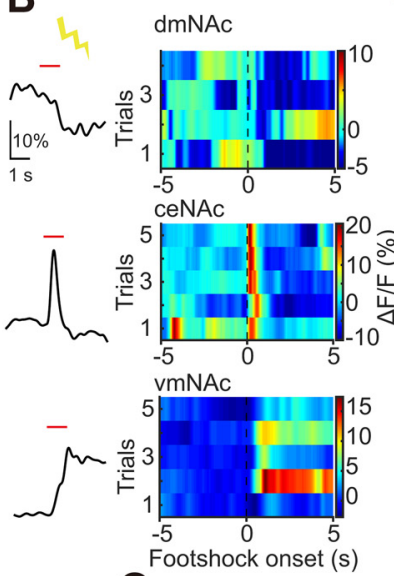

$\mathbf{G}^{\text {Footshock onset (s) }}$

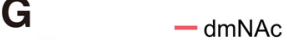

C
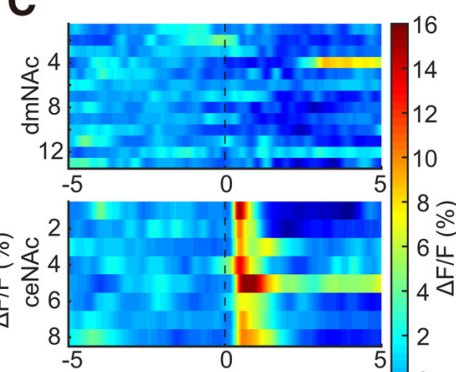

D
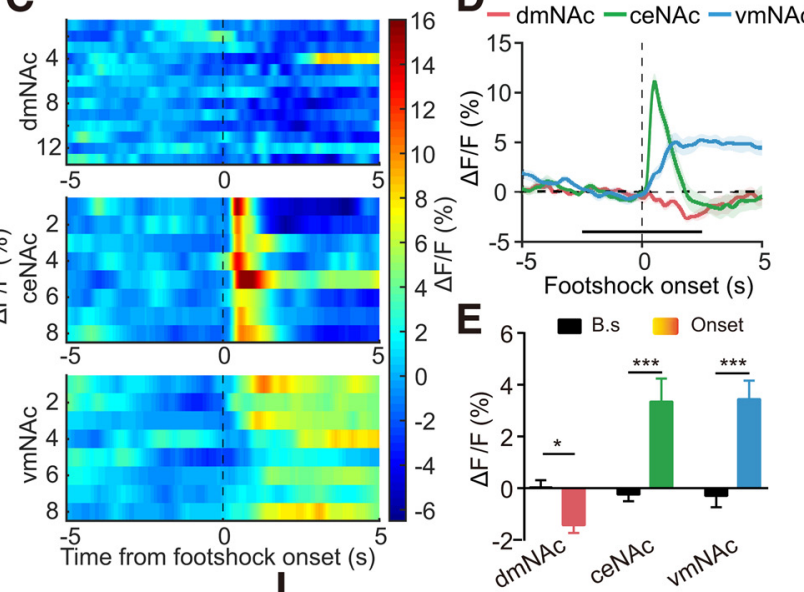

Time from footshock onset (s) I

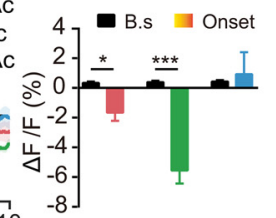

$-\mathrm{dmNAc}-\mathrm{ceNAc}-\mathrm{vmNAc}$
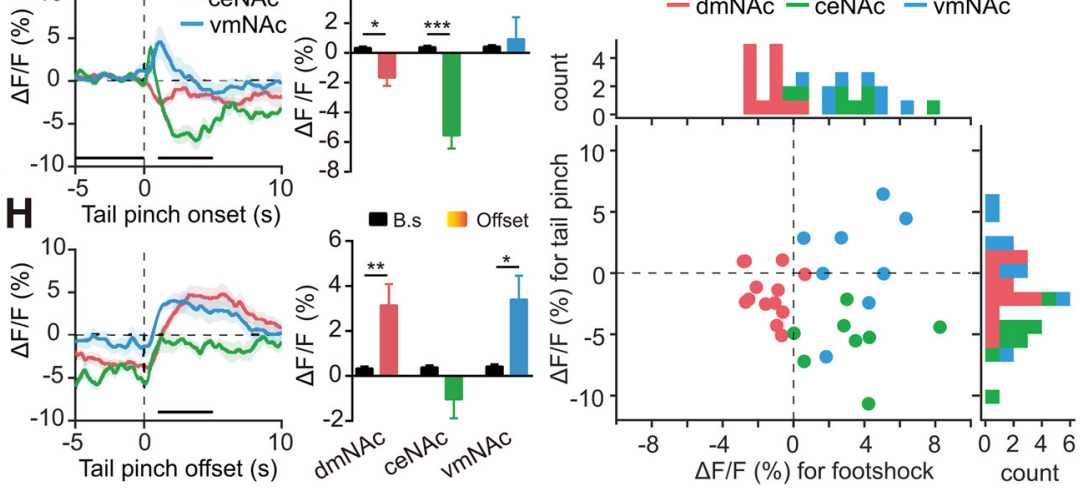

Figure 2. Differential changes of DA-sensor signal in response to unexpectedly noxious stimuli in each NAc subregion. $A$, Schematic of delivery of acute aversive stimuli during fiber photometry recording. $\boldsymbol{B}$, Representative photometry traces in response to footshock (left) and heatmap of dynamics of DA-sensor signal (right) for an example mouse for different subregions of NAc. Each red bar represents a $1 \mathrm{~s}$ footshock event. $C$, Heatmap of averaged change of DA-sensor signal during footshock for individual animals in each group. $D$, Averaged dynamics of DA-sensor signal in each subregion in response to footshock. Black bars represent the baseline period ( -2.5 to $0 \mathrm{~s}, \mathrm{~B} . \mathrm{s})$ and onset period $(0-2.5 \mathrm{~s})$ used for quantification analysis. $E$, Comparison of the averaged DA-sensor signal during B.s and onset period for each group (two-way ANOVA revealed region $\times$ period interaction, $F_{(2,52)}=21.48, p<0.001$; Bonferroni post hoc analysis, ${ }^{*} p=0.0455$ between B.s and onset period for dmNAc region, and ${ }^{* * *} p<0.001$ for ceNAc and vmNAc regions). $F$, Representative photometry traces in response to tail pinch (left) and heatmap of dynamics of DA-sensor signal (right) for an example mouse for each sampling area. Each red bar represents a tail pinch event. G, Left, Averaged dynamics of DA-sensor signal in each subarea across the onset of tail pinch. Black bars represent the baseline period ( -5 to 0 s, B.s) and onset period ( $1-5 \mathrm{~s}$ ) used for quantification analysis. Right, Quantification analysis between the signal during B.s and onset period (two-way ANOVA revealed region $\times$ period interaction, $F_{(2,52)}=10.11, p=0.0002$; Bonferroni post hoc analysis, ${ }^{*} p=0.0475$ between B.s and onset period for dmNAcregion, ${ }^{* * *} p<0.001$ for ceNAcregion and $p>0.9999$ for vmNAcregion). $\boldsymbol{H}$, Averaged dynamics of DA-sensor signal across the offset of tail pinch (left, offset period, $1-5 \mathrm{~s}$ after pinch stop, shown by a black bar) and quantitative analysis (right, two-way ANOVA revealed region $\times$ period interaction, $F_{(2,52)}=5.716, p=0.0057$; Bonferroni post hoc analysis, ${ }^{* *} p=0.0051$ between B.s and offset period for dmNAc region, $p=0.5849$ for ceNAc region and ${ }^{*} p=0.0241$ for vmNAc region). $I$, Joint plot of DA-sensor signal in response to footshock and tail pinch for individual animals in three groups. Histograms represent the distribution of the footshock response (top) or tail pinch response (right). Error bar and shadows represent SEM.

and sweet water consumption and found that the DA-sensor signal was significantly decreased by bitter water in both the dlatNAc and vlatNAc (Fig. 3C), except for the water in the dlatNAc. To further determine the timing that DA-sensor signal exhibited differential dynamics during different liquid consumption, we aligned the DA-sensor signal to the offset of drinking behavior. We found that the DA-sensor signal already decreased to the baseline level at the end of bitter water drinking while the DA level mainly remained above the baseline level for water or sweet water drinking (Fig. 3D). As bitter water might shorten drinking duration, the quick decrease of DA-sensor signal in the bitter water group could be due to short drinking behavior. Therefore, we calculated the averaged drinking duration for each taste and found that there was no significant difference between each group, although the drinking duration indeed showed a decreased tendency for the bitter water group (Fig. 3E). To further minimize the effect of differential drinking duration on quantifying the change of DA-sensor signal, we analyzed the averaged signal within consumption behavior and found that the signal was significantly smaller during bitter water stimuli than that during water or sweet water consumption (Fig. $3 F$ ).

We next further examined whether other aversive stimuli would uniformly decrease DA-sensor signal in the two subregions by challenging mice with footshock during photometry recording. Surprisingly, we found that the DA-sensor signal was decreased by the footshock in the dlatNAc, whereas the DAsensor signal was increased in the vlatNAc (Fig. $3 G-I$ ). As footshock usually evoked jumping behavior, the DA dynamics might include movement artifacts. To rule out this possibility, we expressed DA-insensitive control sensors (DA-sensor-mut) in the dlatNAc and vlatNAc (Sun et al., 2018), whose fluorescence signal should reflect motion effect. We found that the fluorescence of DA-sensor-mut was not significantly changed by footshock stimulus (Fig. 3G,I), suggesting that the change of DA-sensor signal was not caused by movement artifacts. Together, these results revealed that aversive taste and footshock uniformly inhibited the DA release in the dlatNAc but evoked an opposite response in the vlatNAc. 


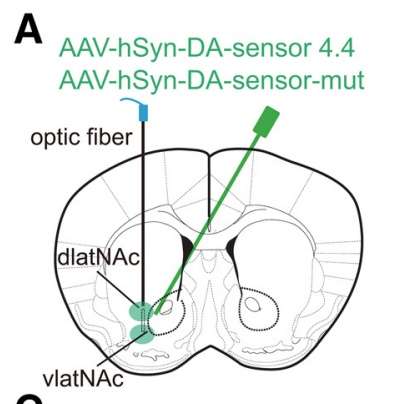

C
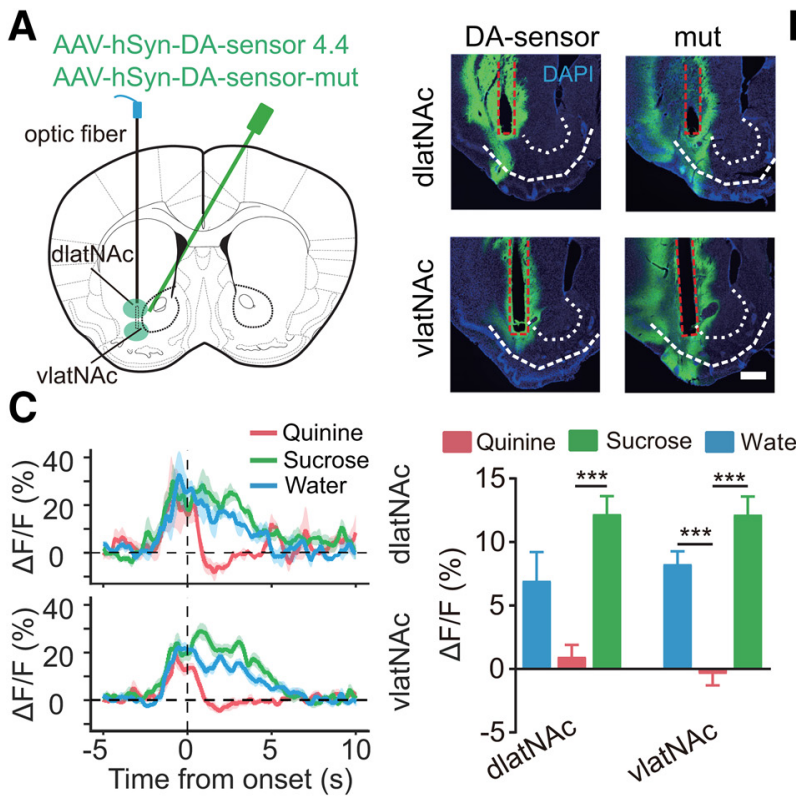

G

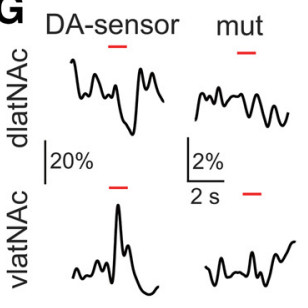

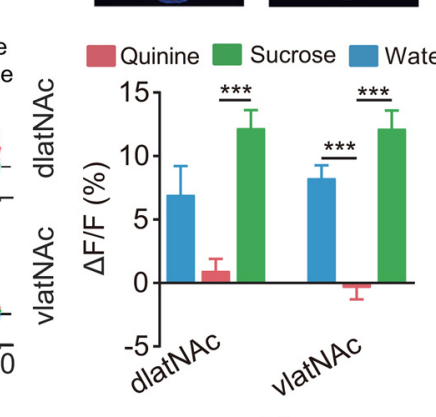

$\mathrm{H}$

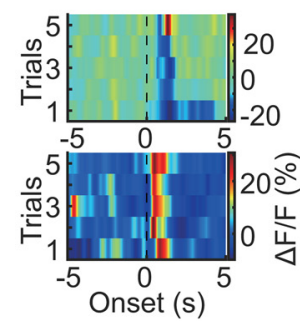

B

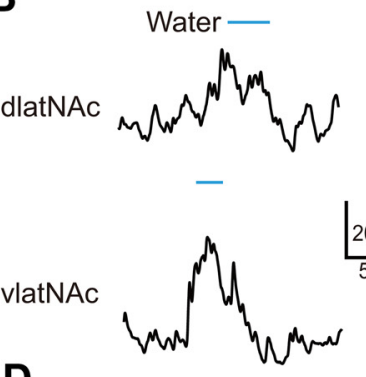

D

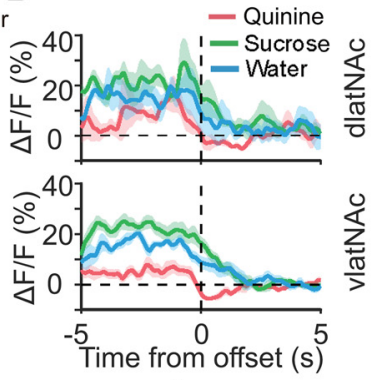

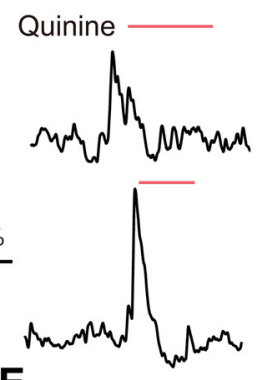

E
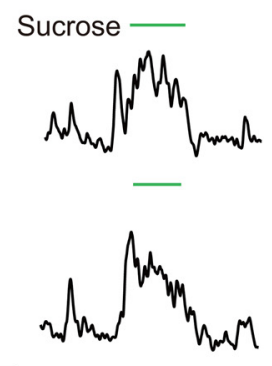

$F$
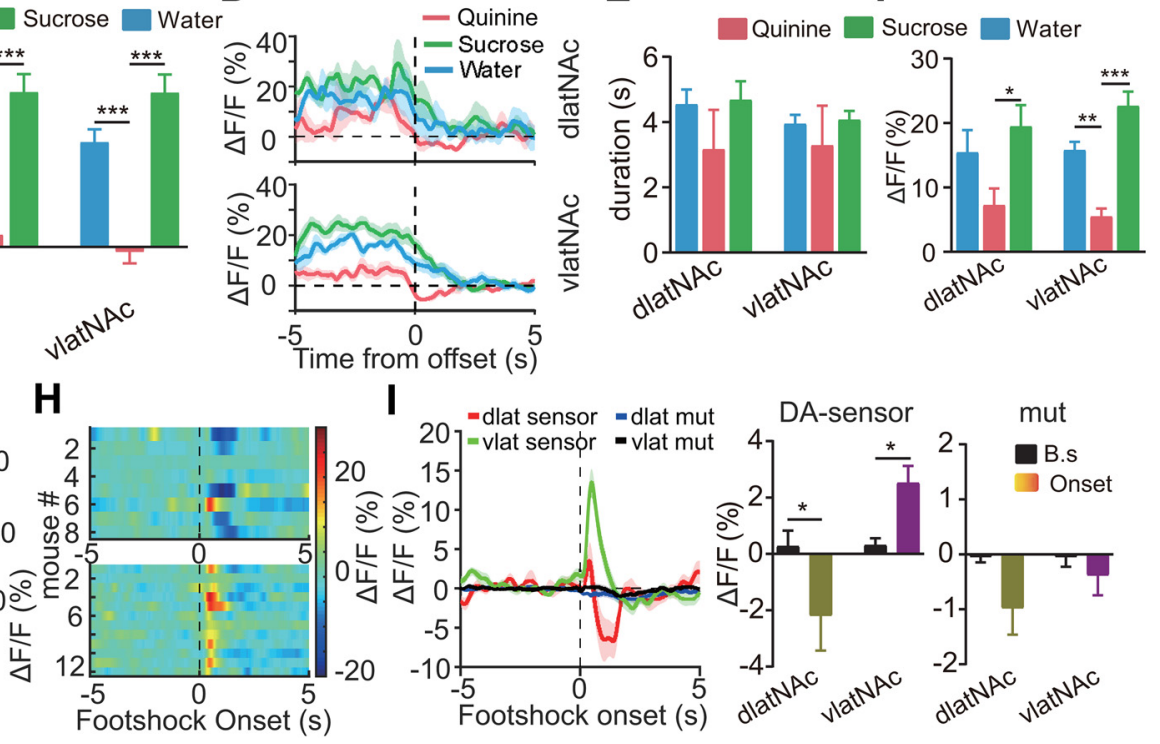

Figure 3. Differential changes of DA sensor-signal in response to liquid consumption and noxious footshock in the ventral and dorsal lateral shell of NAc. $A$, Schematic of virus injection and optic fiber implantation into the dorsal ( $n=8$ mice for DA-sensor and $n=5$ mice for DA-sensor-mut) or ventral ( $n=12$ mice for DA-sensor and $n=5$ mice for DA-sensor-mut) lateral shell of NAc in WT animals (left) and example recording sites in each subarea (right). Dashed red line indicates the track of fiber optic. Scale bar, $500 \mu \mathrm{m}$. $\boldsymbol{B}$, Representative photometry traces during liquid consumption from an example mouse in each subregion. Color bar represents the licking/drinking event. $C$, Dynamics of averaged fluorescence change in response to liquid consumption in each subarea (left) and quantitative comparison (right, two-way ANOVA revealed no region $\times$ taste interaction, $F_{(2,30)}=0.4398, p=0.6482 ;$ Bonferroni post hoc analysis, $p=0.086$ between water and quinine and ${ }^{* * *} p=0.0002$ between sucrose and quinine for dlatNAc region, $n=4$ mice, ${ }^{* * *} p<0.001$ between water or sucrose and quinine for vlatNAc region, $n=8$ mice, and $p>0.13$ for other comparisons). $\boldsymbol{D}$, Dynamics of averaged fluorescence change in response to the end of liquid consumption in each subarea. $\boldsymbol{E}$, Averaged drinking/licking duration for each taste in each subarea (two-way ANOVA revealed no region $\times$ taste interaction, $F_{(2,30)}=0.1190, p=0.8882$; Bonferroni post hoc analysis, $p>0.85$ for all comparisons). $F$, Averaged fluorescence signal within drinking/licking behavior for each taste in each subarea (right, two-way ANOVA revealed no region $\times$ taste interaction, $F_{(2,30)}=0.5955, p=0.5577 ;$ Bonferroni $p$ ost hoc analysis, $p=0.2154$ between water and quinine and ${ }^{*} p=0.0155$ between sucrose and quinine for dlatNAc region, ${ }^{* *} p=0.0028$ between water and quinine and ${ }^{* * *} p<0.001$ between sucrose and quinine for vlatNAc region, and $p>0.08$ for other comparisons). $\boldsymbol{G}$, Representative photometry traces across footshock delivery (left) and dynamics of DA-sensor in each trial (right) for a sensor example mouse in each subarea. $\boldsymbol{H}$, Heatmap of averaged change of DA-sensor fluorescence during footshock for individual animals in dlatNAc (top) and vlatNAc (bottom) group. I Averaged fluorescence dynamics of DA-sensor or sensor-mut in each subregion in response to footshock (left) and comparison of the averaged signal between $B$.s and onset period for each sensor group (middle, two-way ANOVA revealed region $\times$ period interaction, $F_{(1,36)}=12.1, p=$ 0.0013; Bonferroni post hoc analysis, ${ }^{*} p=0.0459$ for dlatNAc region, $n=8$ mice, and ${ }^{*} p=0.0275$ for vlatNAc region, $n=12$ mice) or mut group (right, two-way ANOVA revealed no region $\times$ period interaction, $F_{(1,16)}=0.8924, p=0.3589$; Bonferroni post hoc analysis, $p=0.1074$ for dlatNAc region, and $p=0.9321$ for vlatNAc region). Error bar and shadows represent $S E M$.

\section{Dynamics of DA fiber activity during appetite and aversive events recapitulated the changes in DA-sensor signal in the NAc}

Although DA-sensor-based photometry recording is well suited to specifically detect the dynamics of DA release, it is possible that the DA signal in targeting areas was directly controlled by local excitatory synapses on the presynaptic DA terminals rather than the activity of DA projections itself (Giorguieff et al., 1977). To verify the involvement of the activity of projection-specific DA fibers in encoding rewarding and aversive signal, we injected a Cre-dependent AAV (AAV-hSyn-DIO-GCaMP6s) unilaterally into the VTA of transgenic mice expressing Cre recombinase controlled by the promoter of DAT (also called Slc6a3) gene (Fig. $4 A$ ) and implanted optic fibers into three NAc subregions to measure the calcium activity of local DA terminals with fiber photometry (Fig. $4 B$ ). First, we examined the dynamics of DA terminals when mice received taste stimuli and observed elevated response across the liquid consumption in each NAc subregion (Fig. $4 C$ ). Averaged data showed that the activity of DA fibers was significantly increased by water in the dmNAc (Fig. $4 D, E$ ), confirming the encoding of reward by the mesolimbic DA system. By contrast, the aversive bitter taste stimulus induced smaller activity change compared with that induced by water in the dmNAc (Fig. $4 F, G$ ), supporting the value coding by the DA system in the dmNAc. The sweet taste did not further change the response compared with the water group (Fig. $4 G$ ), consistent with the results obtained by DA-sensor (Fig. 1G).

We next explored whether DA fibers in distinct NAc subregions would be differentially affected by noxious stimuli. We first challenged mice with unexpected footshock stimulus, and found decreased response of DA fibers in the dmNAc and increased response in the vmNAc and ceNAc across different animals (Fig. $4 H$ ). The averaged dynamics of DA fibers and quantitative changes across the onset of footshock stimulus confirmed the 
A
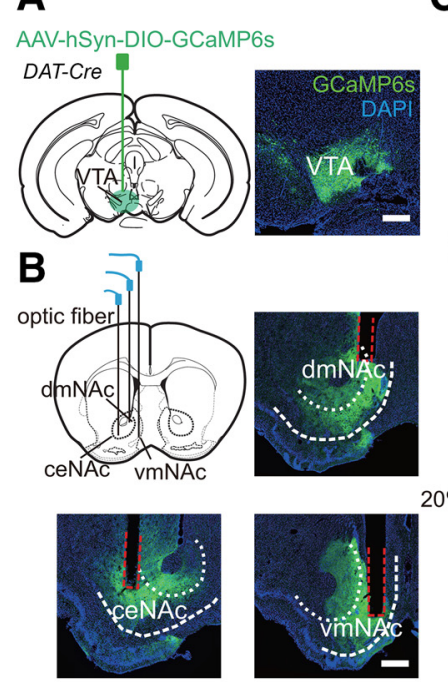

C

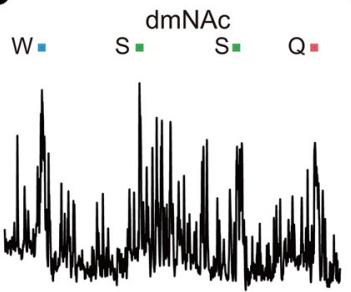

ceNAc
D

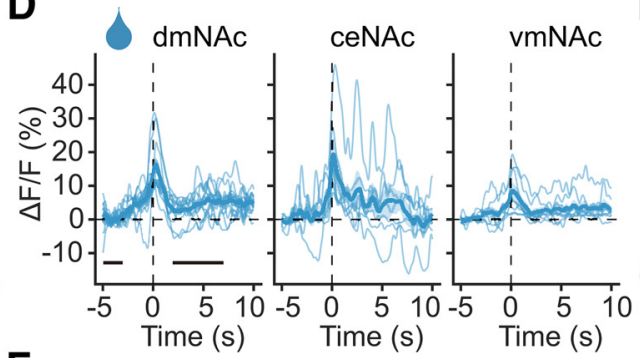

E

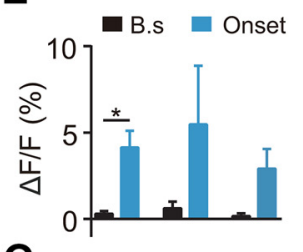

G

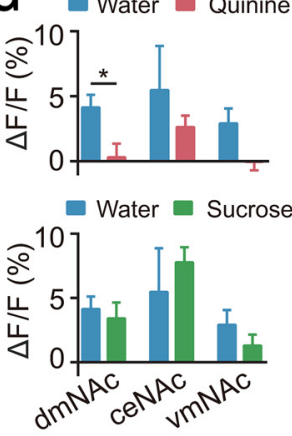

H
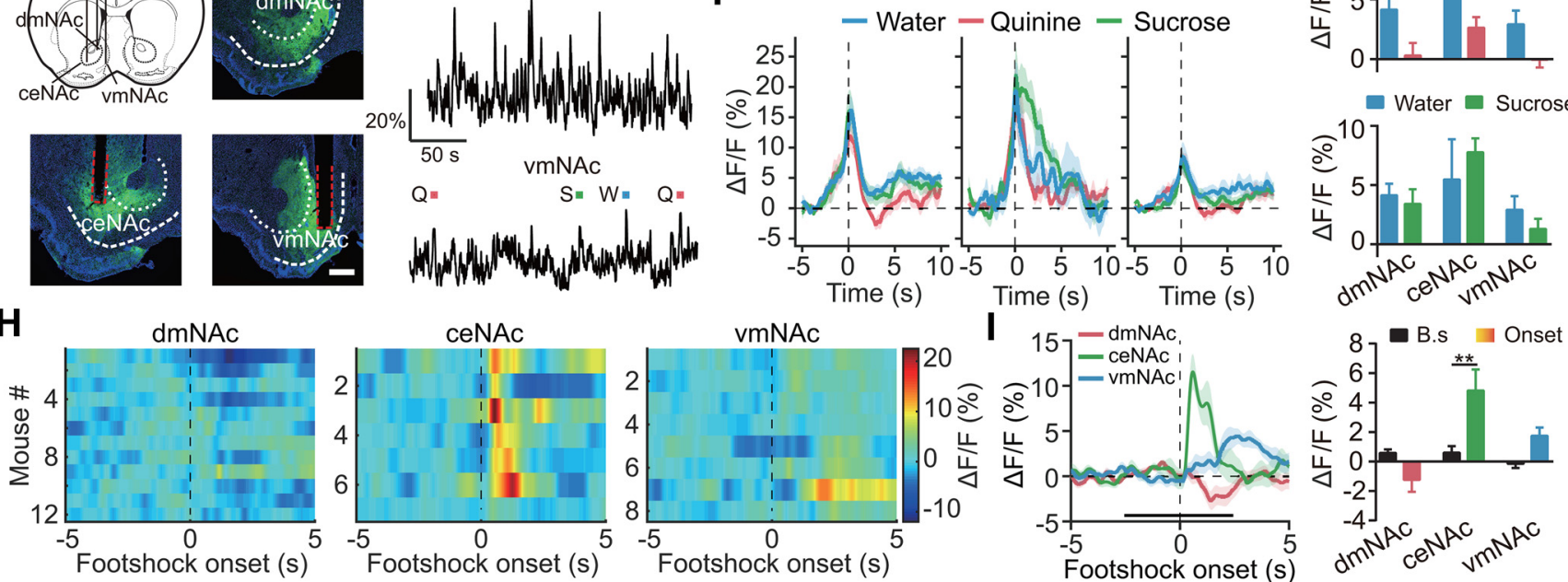

J
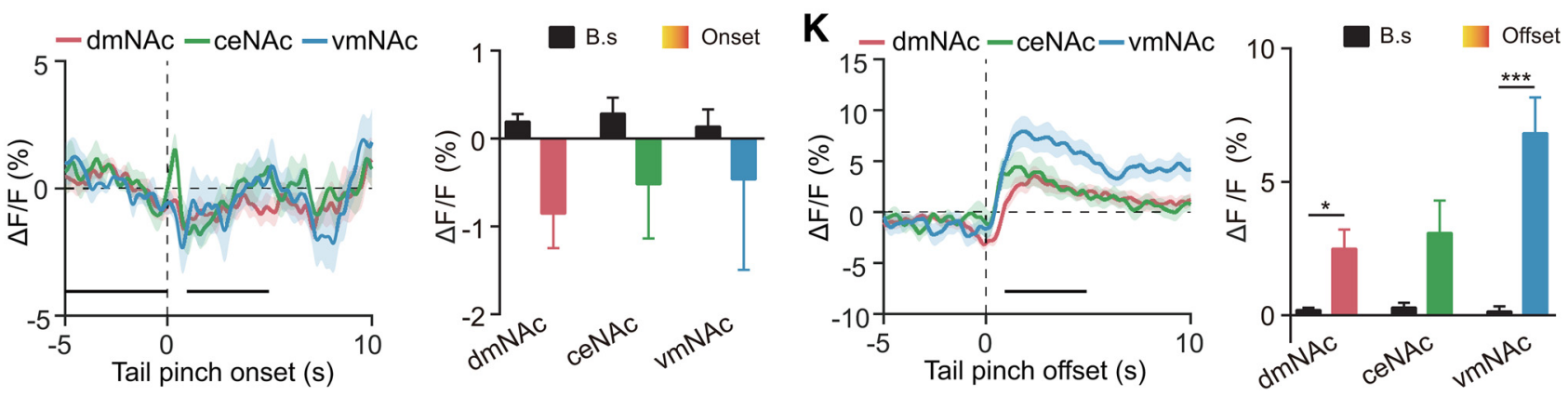

Figure 4. VTA DA fibers in the NAc shell showed diverse response to rewarding and aversive stimulus in a region-specific manner. $A$, Schematic of delivery of AAV-expressing GCaMP6s in the VTA and example of GCaMPGs expression. Scale bar, $500 \mu \mathrm{m}$. B, Schematic of optic fiber implantation in the NAc for recording the activity of DA projection and example of GCaMP6s ${ }^{+}$fibers in each subregion. Scale bar, $500 \mu \mathrm{m}$. C, Representative photometry traces showing the induced transients of DA fibers by liquid consumption in each group. W, Water; $Q$, water with quinine; $S$, water with sucrose. D, Fluorescent change of DA fibers across water consumption in dmNAc group (left, $n=12$ mice), ceNAc group (middle, $n=7$ mice), and vmNAc group (right, $n=8$ mice). Thin lines indicate the response in individual animals. E, Quantitative analysis of GCaMP6s fluorescence change in response to water consumption for each group (two-way ANOVA revealed no region $\times$ period interaction, $F_{(2,48)}=0.3018, p=0.7409$; Bonferroni post hoc analysis, ${ }^{*} p=0.0331$ between B.s and onset period for dmNAc region, $p=0.0626$ for ceNAc region, and $p=0.4633$ for vmNAc region). $F$, Averaged dynamics of DA fibers across $W, S$, and $Q$ consumption for each area. $G$, Quantitative comparison of the difference between the response to W and $Q$ (top, two-way ANOVA revealed no region $\times$ taste interaction, $F_{(2,48)}=0.1816, p=0.8308$; Bonferroni post hoc analysis, ${ }^{*} p=0.0483$ between water and quinine for $\mathrm{dmNA}$ c region, $p=0.6476$ for ceNAc region, and $p=$ 0.5079 for vmNAc region) or $S$ (bottom, two-way ANOVA revealed no region $\times$ taste interaction, $F_{(2,48)}=0.8170, p=0.4478$; Bonferroni post hoc analysis, $p>0.9999$ between water and sucrose for all regions) consumption for each subarea. $\boldsymbol{H}$, Heatmap of dynamics of DA terminals across footshock delivery for individual animals in each group. $\boldsymbol{I}$, Averaged dynamics of DA terminals during footshock (left) and quantitative comparison (right, two-way ANOVA revealed region $\times$ period interaction, $F_{(2,48)}=9.780, p=0.0003$; Bonferroni post hoc analysis, $p=0.1160$ between B.s and onset period for dmNAc region, ${ }^{* *} p=0.0015$ for ceNAc region, and $p=0.2356$ for vmNAc region). $J$, Averaged dynamics of DA terminals across tail pinch onset (left) and quantitative comparison (right, two-way ANOVA revealed no region $\times$ period interaction, $F_{(2,48)}=0.1167, p=0.8901$; Bonferroni post hoc analysis, $p=0.2488$ between B.s and onset period for dmNAc region, $p=0.9151$ for ceNAc region, and $p>0.9999$ for vmNAc region). $K$, Averaged dynamics of DA terminals across tail pinch offset (left) and quantitative comparison (right, two-way ANOVA revealed region $\times$ period interaction, $F_{(2,48)}=5.058, p=0.0102$; Bonferroni post hoc analysis, ${ }^{*} p=0.0453$ between B.s and offset period for dmNAc region, $p=0.0693$ for ceNAc region, and ${ }^{* * *} p<0.001$ for vmNAc region). Error bar and shadows represent SEM.

significant change in the ceNAc (Fig. 4I), similar to the response of DA sensor (Fig. 2D,E). Finally, we delivered acute tail pinch stimulus to the animals and found that the activity of DA fibers was not significantly affected after the onset of stimuli (Fig. $4 J$ ), although the activity showed a decreasing tendency. For the response after the cession of aversive tail pinch, we found that the activity of DA fibers in the dmNAc and vmNAc was significantly elevated (Fig. 4K). In summary, the rewarding and aversive stim- uli excited and inhibited DA fibers in the dmNAc respectively, whereas these salient stimuli predominantly excited the DA fibers in the vmNAc.

Encoding of reward and aversion by the mesolimbic DA system was related to the DV axis of the NAc

Our recording result showed that the response of the DA system to rewarding and aversive stimuli was heterogeneous in different 


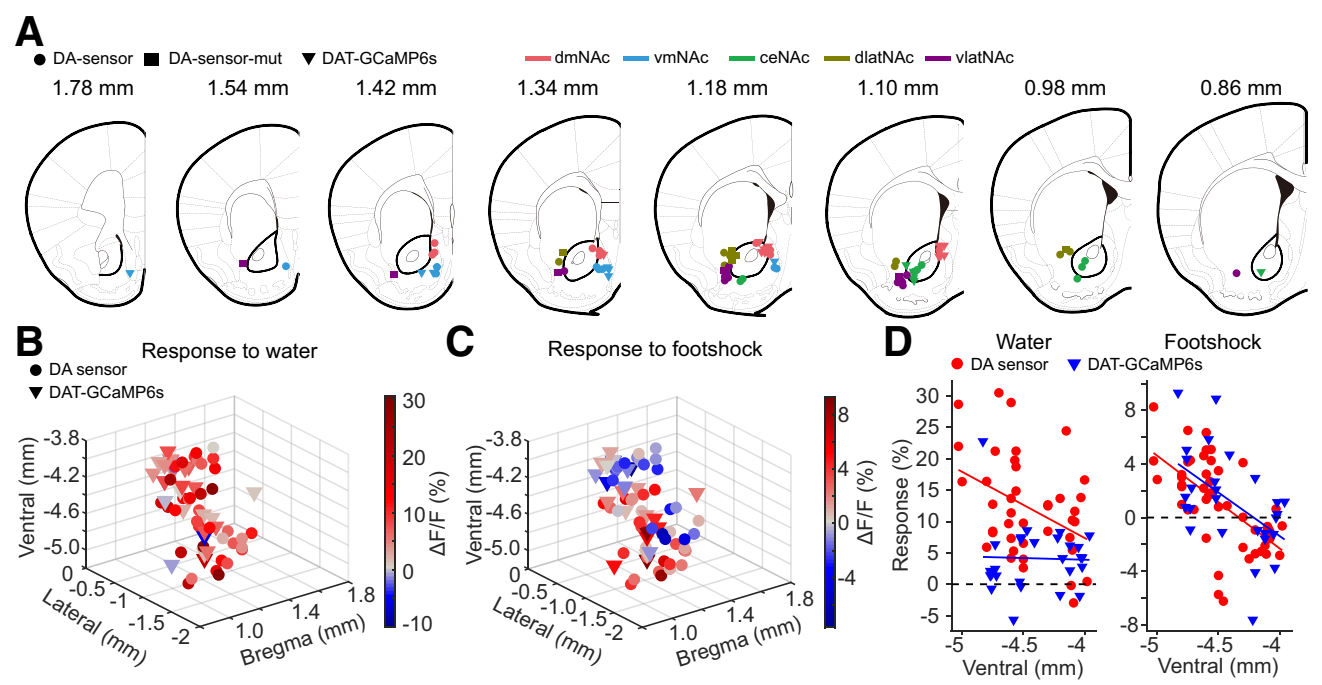

Figure 5. Transitional encoding of value to salience by mesolimbic DA system along the DV axis of NAc. $A$, The location of optic fiber tips for all DA-sensor, DA-sensor-mut, and DAT-GCaMP6s animals. $\boldsymbol{B}$, The topographic heatmap of response to water for the individual animals. $\boldsymbol{C}$, The topographic heatmap of response to footshock for the individual animals. $\boldsymbol{D}$, Correlation analysis of the relationship between the depth of sampling site and the magnitude of response to water consumption (left, Pearson's correlation test, $p=0.0119$ for DA-sensor animals and $p=0.8785$ for GCaMP6s animals) and footshock stimulus (right, Pearson's correlation test, $p<0.001$ for DA-sensor animals and $p=0.005$ for GCaMP6s animals).

NAc subregions. To quantitatively analyze the relationship between neural response and its anatomical location, we verified the recording sites for all the mice used for recording (Fig. 5A). We next visualized the response of DA sensor or DA fibers to appetite water stimuli (Fig. 5B) on the recording location map and found that rewarding taste stimuli evoked robust increase of DA signal recorded by both DA-sensor and GCaMP6s across the NAc. In contrast, aversive footshock dominantly evoked an inhibitory response in the dorsal part of NAc shell (including dlatNAc and dmNAc) and excitatory response in the ventral NAc shell (including vlatNAc and vmNAc) (Fig. 5C). We next directly tested the correlation between the response of DA-sensor/fibers signal to water or footshock stimuli and the depth of sampling sites and found that the response magnitude significantly changed along the axis from dorsal to ventral NAc, except for the response to water in the GCaMP6s group (Fig. 5D). Together, these results reveal that the DA system gradually changed their encoding property from encoding value to salience signal in the dorsal to ventral axis.

\section{Discussion}

By measuring the release of DA in different subregions of NAc, we found that the DA system in the dmNAc and dlatNAc encodes the value signal, whereas that in the vmNAc mainly encodes salience signal. In contrast, the DA system in the ceNAc and vlatNAc might encode both value and salience signal. This is further supported by the results obtained by measuring the activity dynamics of DA fibers in different NAc subregions. These results uncovered a comprehensive encoding map of diverse reward and aversive events by the mesolimbic DA system, consistent with a recent study (de Jong et al., 2019).

Our results showed that the release of DA or the activity of DA fibers was uniformly increased by rewarding water/sweet water stimulus across all subregions of NAc (Figs. 1E, 3C, 4E), consistent with the critical role of the mesolimbic DA system in processing reward information (Björklund and Dunnett, 2007; Berridge and Kringelbach, 2015; Hamid et al., 2016; Howe and Dombeck, 2016; Saunders et al., 2018). The DA level increased before reward consumption and peaked at the onset of consump- tion behavior (Figs. $1 D, 3 C$ ), consistent with a previous study showing that DA release peaked near the onset of lever press for sucrose reward determined by cyclic voltammetry (Cameron et al., 2014), supporting the involvement of the mesolimbic DA system in motivating subjects to seek reward. The response to water was not further enhanced by sweet taste (Figs. $1 G, 3 C, 4 G$ ), partially because the water was already a big reward for waterrestricted animals or DA signals in the ventral striatum do not discriminate reward size in freely moving animals very well (Saddoris et al., 2017). Interestingly, the release of DA and the activity of DA fibers also increased at the offset of tail pinch across all NAc subregions (Figs. $2 \mathrm{H}, 4 \mathrm{~K}$ ), although not significantly in the ceNAc, consistent with the induction of DA release after the cession of aversive stimuli in previous studies (Seymour et al., 2005; Budygin et al., 2012; de Jong et al., 2019). The termination of an aversive event might be rewarding and DA is found to be critical for the generation of preference for the environment associated with pain relief (Navratilova et al., 2015). Therefore, the increased release of DA at the offset of tail pinch might represent reward generated by the relief of aversive stimuli.

The response of the mesolimbic DA system to aversive stimuli was critically associated with the subregions of the NAc. Specifically, aversive bitter taste, footshock, and tail pinch dominantly decreased the release of DA or the activity of DA axons in the dorsal NAc shell (including dmNAc and dlatNAc) yet evoked elevation in the release of DA or the activity of DA axons in the vmNAc (Figs. 1G, 2E, G, 3C,I, 4G). These results are consistent with a recent photometry study showing that footshock suppressed and excited the activity of DA fibers in the lateral NAc shell and the vmNAc shell (de Jong et al., 2019), which resemble the dlatNAc and vmNAc/ceNAc in our study, respectively. Our result suggests that various aversive events might be encoded in a similar way within the dmNAc, dlatNAc, or vmNAc itself. Furthermore, our data indicate that the DA system in the dmNAc and dlatNAc encodes the value of the stimuli, and that, in the vmNAc, encodes the salience. The finding that aversive events evoked transitional DA changes along DV NAc (Fig. 5C,D) could explain some inconsistent results in previous studies. For exam- 
ple, a study reported that footshock paired context elicited DA release in both the NAc core and shell by microdialysis probe recording (Martinez et al., 2008). After examining the recording site in this study, we found that the sampling in the NAc core actually centered in the ventral NAc core, close to the vmNAc in our study, in which DA-sensor signal was also increased by footshock. Previous studies showed that NAc is quite heterogeneous; for example, opioids and glutamate differentially affected rewarding and aversive events from dorsal to ventral NAc or from rostral to caudal NAc (Castro and Berridge, 2014; Al-Hasani et al., 2015). The function of DA in distinct NAc subregions was found to be related to its differential interaction with glutamatergic circuits via diverse DA receptors (Faure et al., 2008; Richard and Berridge, 2011). Our result showing that the value and salience signals are separately encoded by the DA system in the dmNAc/dlatNAc and vmNAc (Fig. $5 B-D$ ) further emphasizes the heterogeneity of the mesolimbic DA system and supports the notion that the hedonic hotspot is finely embedded in a specific subregion of the limbic area (Seymour et al., 2007; Berridge and Kringelbach, 2015).

One interesting finding in our recording was that the information of reward and aversion is not simply encoded in either value or salience way by the DA system in the ceNAc and vlatNAc. Both rewarding water and aversive footshock increased the DAsensor signal or the activity of DA fibers (Figs. 1E, 2E, 3I, $4 I$ ) in the ceNAc and vlatNAc, whereas aversive bitter taste and tail pinch decreased the DA-sensor signal in these areas (Figs. 1G, 2G, $3 C)$. The similar response pattern to rewarding and aversive stimuli in the ceNAc and vlatNAc might be due to the fact that the recording sites were very close to each other (Fig. $5 \mathrm{~A}$ ), suggesting that they might receive similar inputs. Previous studies found that optogenetic activation of inputs to the VTA from the laterodorsal tegmentum evoked conditioned place preference, which requires the DA receptors in the NAc lateral shell (Lammel et al., 2012). Our results extended the function of DA pathway from VTA to the NAc lateral shell by revealing that the DA signal in the vlatNAc encodes both value and salience signature, probably in a stimuli-dependent manner, whereas the DA signal in the dlatNAc encodes value signal.

The results obtained by the fluorescent DA-sensor and the calcium indicator GCaMP6s showed slight differences. For example, bitter water induced a decrease of response in the ceNAc during the recording using DA-sensor but no significant alteration using GCaMP6s (Fig. $1 G$ vs Fig. $4 G$ ). One reason might be that DA axons passing through the sampling site may have contributed to the GCaMP6s signal, which was unrelated to the activity of local DA projections (Giorguieff et al., 1977), although calcium transients in passing axons were found to be smaller than that in axon terminals (Koester and Sakmann, 2000). Another possibility is that the GCaMP6s signal correlated to DA release was mixed with the signal corresponding to glutamate release in the DA fibers because some DA neurons projecting to the ventral striatum are able to corelease glutamate (Stuber et al., 2010). The technique limitation of specifically recording DA projection with GCaMP6s emphasizes the importance of recording using the fluorescent DA-sensor. Therefore, complementary approaches will provide a more comprehensive view of DA dynamics.

In conclusion, our results indicate that the mesolimbic DA system in the dmNAc/dlatNAc, vmNAc, and ceNAc/vlatNAc mainly encodes value, salience, and mixture of value and salience signal, respectively. These results extend our understanding of the functional topography of the mesolimbic DA system and pro- vide important insight into how the mesolimbic DA system processes motivational information.

\section{References}

Al-Hasani R, McCall JG, Shin G, Gomez AM, Schmitz GP, Bernardi JM, Pyo CO, Park SI, Marcinkiewcz CM, Crowley NA, Krashes MJ, Lowell BB, Kash TL, Rogers JA, Bruchas MR (2015) Distinct subpopulations of nucleus accumbens dynorphin neurons drive aversion and reward. Neuron 87:1063-1077.

Anstrom KK, Miczek KA, Budygin EA (2009) Increased phasic dopamine signaling in the mesolimbic pathway during social defeat in rats. Neuroscience 161:3-12.

Badrinarayan A, Wescott SA, Vander Weele CM, Saunders BT, Couturier BE, Maren S, Aragona BJ (2012) Aversive stimuli differentially modulate real-time dopamine transmission dynamics within the nucleus accumbens core and shell. J Neurosci 32:15779-15790.

Bassareo V, De Luca MA, Di Chiara G (2002) Differential expression of motivational stimulus properties by dopamine in nucleus accumbens shell versus core and prefrontal cortex. J Neurosci 22:4709-4719.

Berridge KC, Kringelbach ML (2015) Pleasure systems in the brain. Neuron 86:646-664.

Björklund A, Dunnett SB (2007) Dopamine neuron systems in the brain: an update. Trends Neurosci 30:194-202.

Brischoux F, Chakraborty S, Brierley DI, Ungless MA (2009) Phasic excitation of dopamine neurons in ventral VTA by noxious stimuli. Proc Nat Acad Sci U S A 106:4894-4899.

Bromberg-Martin ES, Matsumoto M, Hikosaka O (2010) Dopamine in motivational control: rewarding, aversive, and alerting. Neuron 68:815-834.

Budygin EA, Park J, Bass CE, Grinevich VP, Bonin KD, Wightman RM (2012) Aversive stimulus differentially triggers subsecond dopamine release in reward regions. Neuroscience 201:331-337.

Cameron CM, Wightman RM, Carelli RM (2014) Dynamics of rapid dopamine release in the nucleus accumbens during goal-directed behaviors for cocaine versus natural rewards. Neuropharmacology 86:319-328.

Castro DC, Berridge KC (2014) Opioid hedonic hotspot in nucleus accumbens shell: mu, delta, and kappa maps for enhancement of sweetness "liking” and "wanting." J Neurosci 34:4239-4250.

Cohen JY, Haesler S, Vong L, Lowell BB, Uchida N (2012) Neuron-typespecific signals for reward and punishment in the ventral tegmental area. Nature 482:85-88.

de Jong JW, Afjei SA, Pollak Dorocic I, Peck JR, Liu C, Kim CK, Tian L, Deisseroth K, Lammel S (2019) A neural circuit mechanism for encoding aversive stimuli in the mesolimbic dopamine system. Neuron 101: 133-151.e7.

Faure A, Reynolds SM, Richard JM, Berridge KC (2008) Mesolimbic dopamine in desire and dread: enabling motivation to be generated by localized glutamate disruptions in nucleus accumbens. J Neurosci 28: 7184-7192.

Fiorillo CD (2013) Two dimensions of value: dopamine neurons represent reward but not aversiveness. Science 341:546-549.

Gantz SC, Ford CP, Morikawa H, Williams JT (2018) The evolving understanding of dopamine neurons in the substantia nigra and ventral tegmental area. Annu Rev Physiol 80:219-241.

Gerber B, Yarali A, Diegelmann S, Wotjak CT, Pauli P, Fendt M (2014) Pain-relief learning in flies, rats, and man: basic research and applied perspectives. Learn Mem 21:232-252.

Giorguieff MF, Kemel ML, Glowinski J (1977) Presynaptic effect of L-glutamic acid on the release of dopamine in rat striatal slices. Neurosci Lett 6:73-77.

Hamid AA, Pettibone JR, Mabrouk OS, Hetrick VL, Schmidt R, Vander Weele CM, Kennedy RT, Aragona BJ, Berke JD (2016) Mesolimbic dopamine signals the value of work. Nat Neurosci 19:117-126.

Howe MW, Dombeck DA (2016) Rapid signalling in distinct dopaminergic axons during locomotion and reward. Nature 535:505-510.

Koester HJ, Sakmann B (2000) Calcium dynamics associated with action potentials in single nerve terminals of pyramidal cells in layer $2 / 3$ of the young rat neocortex. J Physiol 529:625-646.

Lammel S, Ion DI, Roeper J, Malenka RC (2011) Projection-specific modulation of dopamine neuron synapses by aversive and rewarding stimuli. Neuron 70:855-862.

Lammel S, Lim BK, Ran C, Huang KW, Betley MJ, Tye KM, Deisseroth K, 
Malenka RC (2012) Input-specific control of reward and aversion in the ventral tegmental area. Nature 491:212-217.

Martinez RC, Oliveira AR, Macedo CE, Molina VA, Brandão ML (2008) Involvement of dopaminergic mechanisms in the nucleus accumbens core and shell subregions in the expression of fear conditioning. Neurosci Lett 446:112-116.

Matsumoto H, Tian J, Uchida N, Watabe-Uchida M (2016) Midbrain dopamine neurons signal aversion in a reward-context-dependent manner. eLife 5:e17328.

Matsumoto M, Hikosaka O (2009) Two types of dopamine neuron distinctly convey positive and negative motivational signals. Nature 459: 837-841.

McCutcheon JE, Ebner SR, Loriaux AL, Roitman MF (2012) Encoding of aversion by dopamine and the nucleus accumbens. Front Neurosci 6:137.

Morales M, Margolis EB (2017) Ventral tegmental area: cellular heterogeneity, connectivity and behaviour. Nat Rev Neurosci 18:73-85.

Navratilova E, Atcherley CW, Porreca F (2015) Brain circuits encoding reward from pain relief. Trends Neurosci 38:741-750.

Parker NF, Cameron CM, Taliaferro JP, Lee J, Choi JY, Davidson TJ, Daw ND, Witten IB (2016) Reward and choice encoding in terminals of midbrain dopamine neurons depends on striatal target. Nat Neurosci 19:845-854.

Patriarchi T, Cho JR, Merten K, Howe MW, Marley A, Xiong WH, Folk RW, Broussard GJ, Liang R, Jang MJ, Zhong H, Dombeck D, von Zastrow M, Nimmerjahn A, Gradinaru V, Williams JT, Tian L (2018) Ultrafast neuronal imaging of dopamine dynamics with designed genetically encoded sensors. Science 360:eaat 4422 .

Richard JM, Berridge KC (2011) Nucleus accumbens dopamine/glutamate interaction switches modes to generate desire versus dread: $\mathrm{D}(1)$ alone for appetitive eating but $\mathrm{D}(1)$ and $\mathrm{D}(2)$ together for fear. J Neurosci 31:12866-12879.

Saddoris MP, Sugam JA, Carelli RM (2017) Prior cocaine experience impairs normal phasic dopamine signals of reward value in accumbens shell. Neuropsychopharmacology 42:766-773.
Salamone JD, Correa M (2012) The mysterious motivational functions of mesolimbic dopamine. Neuron 76:470-485.

Saunders BT, Richard JM, Margolis EB, Janak PH (2018) Dopamine neurons create Pavlovian conditioned stimuli with circuit-defined motivational properties. Nat Neurosci 21:1072-1083.

Schultz W (2006) Behavioral theories and the neurophysiology of reward. Annu Rev Psychol 57:87-115.

Schultz W (2016) Dopamine reward prediction-error signalling: a twocomponent response. Nat Rev Neurosci 17:183-195.

Schultz W, Dayan P, Montague PR (1997) A neural substrate of prediction and reward. Science 275:1593-1599.

Seymour B, O’Doherty JP, Koltzenburg M, Wiech K, Frackowiak R, Friston K, Dolan R (2005) Opponent appetitive-aversive neural processes underlie predictive learning of pain relief. Nat Neurosci 8:1234-1240.

Seymour B, Daw N, Dayan P, Singer T, Dolan R (2007) Differential encoding of losses and gains in the human striatum. J Neurosci 27:4826-4831

Stuber GD, Hnasko TS, Britt JP, Edwards RH, Bonci A (2010) Dopaminergic terminals in the nucleus accumbens but not the dorsal striatum corelease glutamate. J Neurosci 30:8229-8233.

Sun F, Zeng J, Jing M, Zhou J, Feng J, Owen SF, Luo Y, Li F, Wang H, Yamaguchi T, Yong Z, Gao Y, Peng W, Wang L, Zhang S, Du J, Lin D, Xu M, Kreitzer AC, Cui G, et al. (2018) A genetically encoded fluorescent sensor enables rapid and specific detection of dopamine in flies, fish, and mice. Cell 174:481-496.e19.

Ungless MA, Magill PJ, Bolam JP (2004) Uniform inhibition of dopamine neurons in the ventral tegmental area by aversive stimuli. Science 303:2040-2042.

Wise RA (2004) Dopamine, learning and motivation. Nat Rev Neurosci 5:483-494.

Yuan L, Liang TY, Deng J, Sun YG (2018) Dynamics and functional role of dopaminergic neurons in the ventral tegmental area during itch processing. J Neurosci 38:9856-9869. 